\title{
Necesidades no materiales en el cuidado de personas con discapacidad intelectual en residencias
}

\section{Non-material needs in the care of people with intellectual disabilities in residences \\ Necessidades não materiais no cuidado de pessoas com deficiência intelectual em residências}

\section{Oscar Martínez-Rivera1 ${ }^{1}$, Enric Benavent Vallès ${ }^{2}$ y Lisette Navarro-Segura ${ }^{3}$}

${ }^{1}$ Doctor. Facultad de Educación social y Trabajo social Pere Tarrés. Universidad Ramon Llull. Correo electrónico: omartinez@peretarres.org

${ }^{2}$ Doctor. Facultad de Educación social y Trabajo social Pere Tarrés. Universidad Ramon Llull. Correo electrónico: ebenavent@peretarres.org

${ }^{3}$ Doctora. Facultad de Educación social y Trabajo social Pere Tarrés. Universidad Ramon Llull. Correo electrónico: lnavarro@peretarres.org

Cómo citar este artículo en edición digital: Martínez-Rivera, O., Benavent Vallès, E. y NavarroSegura, L. (2020). Necesidades no materiales en el cuidado de personas con discapacidad intelectual en residencias. Cultura de los Cuidados (Edición digital), 24 (56) Recuperado de http://dx.doi.org/10.14198/cuid.2020.56.13

Correspondencia: Calle Santaló, 37. 08021 Barcelona (Spain)

Correo electrónico de contacto: omartinez@peretarres.org

Recibido:7/12/2020

Aceptado:12/03/2020

\section{(1)}

\section{ABSTRACT}

There is a broad consensus among care professionals about the importance of the attention to non-material needs. In the care of people with disabilities, these issues acquire a special relevance (Swinton, 2002, Clarke \& Cardman, 2002, Kaye \& Raghavan, 2002). Spirituality helps to face complex situations and has a direct relationship with well-being and resilience.
In the health and social professions, this internal dimension must be kept in mind, either from the point of view of giving meaning to life, or in religious terms. Residential homes, where people with intellectual disabilities live, are ideal places to take care of these aspects. A questionnaire was sent to 172 professionals and the data were triangulated with interviews with family members, professionals and people with intellectual disabilities. The results show that people with disabilities who live in residences make decisions about their 
own lives, especially in the short term. On the other hand, these decisions decrease as it has a more long-term implication. In addition, we perceive residential homes as places where there are spaces of silence related to the interiority of people with intellectual disabilities.

Key words: Non-material needs, disability, residences, spirituality, professionals.

\section{RESUMEN}

Hay un amplio consenso entre los profesionales de la atención a las personas de que la atención a las necesidades no materiales es importante. En el cuidado de personas con discapacidad éstas cuestiones adquieren una relevancia especial (Swinton, 2002; Clarke y Cardman, 2002; Kaye y Raghavan, 2002). La espiritualidad ayuda a afrontar situaciones complejas y tiene relación directa con el bienestar y la resiliencia. En las profesiones sanitarias y sociales esta dimensión interior debe tenerse en cuenta, ya sea desde el punto de vista de dar sentido a la vida o bien en términos religiosos. Las residencias donde viven las personas con discapacidad intelectual son lugares que se prestan a tener en cuenta estos aspectos. Se pasó un cuestionario a 172 profesionales y se han triangulado los datos con entrevistas a familiares, profesionales y personas con discapacidad intelectual. Se constata que las personas con discapacidad que viven en centros residenciales toman decisiones sobre sus propias vidas sobre todo a corto plazo. Por otro lado, estas decisiones disminuyen a medida que tiene una implicación más a largo plazo. Además, los centros residenciales son percibidos como lugares donde se dan espacios de silencio relacionado con la interioridad de las personas con discapacidad intelectual.

Palabras clave: Necesidades no materiales, discapacidad, centros residenciales, espiritualidad, profesionales.

\section{RESUMO}

Entre os profissionais da atenção às pessoas, é consenso que estar atento às necessidades não materiais é importante, visto que elas adquirem relevância especial ao se tratar de pessoas portadoras de deficiência. A espiritualidade ajuda no enfrentamento de situações complexas e tem relação com o bem estar e com a resiliência. As profissões sanitárias e as sociais precisam considerar esta dimensão interior, seja do ponto de vista de dar sentido à vida ou do religioso e, nas residências das pessoas portadoras de deficiência, tais aspectos merecem atenção especial. Aplicou-se um questionário a 172 profissionais e triangularam-se os dados com entrevistas a familiares, profissionais e pessoas portadoras de deficiência intelectual. Constatou-se que pessoas portadoras de deficiência que vivem em centros residenciais decidem sobre suas vidas especialmente a curto prazo. Por outro lado, tais decisões diminuem quando se referem a aspectos de longo prazo. Além disso, evidencia-se a percepção dos centros residenciais como espaços de silêncio, relacionados à interioridade das pessoas portadoras de deficiências intelectuais.

Palavras chaves: Necessidades não materiais, deficiências, centros residenciais, espiritualidade, profissionais

\section{INTRODUCCIÓN}

La determinación por atender las necesidades no materiales de las personas nos sitúa en un ámbito de difícil definición. La gradual disposición de las necesidades que se plantea en algunas taxonomías dibuja un recorrido que va desde lo más estrictamente material hasta lo no material. Las necesidades más específicamente humanas no se encuentran en la base sino en la parte superior de la pirámide, por tanto, hablar de necesidades no materiales es hablar de uno de los aspectos más determinantes del ser humano.

Para abordar las necesidades no materiales debemos acercarnos a conceptos como el de interioridad, de yo profundo y 
Revista científica de la Asociación de Historia y Antropología de los Cuidados (Universidad de Alicante)

también de espiritualidad, entendida como la filosofía de vida que guía la conducta y que se sitúa en el núcleo de la existencia individual (Kaye y Raghavan, 2002). La interioridad, tal como apunta Otón (2018), actúa como una brújula que orienta la dirección por donde debe transcurrir la vida, y por tanto es el ámbito de construcción de sentido.

La interioridad de la persona se refiere pues a una dimensión no corporal y no mental que actúa como fuente de sentido. La vivencia de esta dimensión interior y los comportamientos que se derivan de dicha vivencia es lo que podemos llamar espiritualidad. La espiritualidad así entendida es el núcleo de la existencia individual.

Es importante poder diferenciar la naturaleza espiritual de las personas de su religiosidad. La espiritualidad es un concepto más amplio y transversal que la religiosidad que se refiere a una determinada forma de vivir la espiritualidad caracterizada por elementos visibles, compartidos en una comunidad, como ritos, creencias, valores o formas de conducta.

Senreich (2013, p. 553) propone esta definición inclusiva de la espiritualidad abierta a las diversas posibilidades de concreción de la dimensión espiritual:

La espiritualidad se refiere a una relación subjetiva del ser humano (cognitiva, emocional e intuitiva) con lo que es desconocido de la existencia, y cómo una persona integra esta relación en una perspectiva sobre el universo, el mundo, los otros, uno mismo, los valores morales, y con el propio sentido de la vida. La espiritualidad se describe como un antecedente de la transcendencia, como una relación con el misterio de la propia existencia que invita a contemplar la vida desde una determinada perspectiva.

La espiritualidad también se ha definido en ocasiones (Vanistendal, 2003; Girard, 2007) como recurso importante para hacer frente a situaciones difíciles. Numerosos estudios han mostrado la relación directa entre el bienestar espiritual y la resiliencia (Koening, 2001; JaramilloVélez, Ospina-Muñoz, Cabarcas-Iglesias y Humphreys, 2005). La OMS (1998) pone de relieve que las creencias religiosas o espirituales de las personas pueden ser de ayuda para afrontar las dificultades de su vida brindando una sensación de bienestar.

El rol de la espiritualidad incrementa claramente en personas mayores, enfermas o con alguna adición o discapacidad. La vivencia de la propia dimensión interior puede ser de ayuda para hacer frente a situaciones de estrés, de frustración o de dolor.

La espiritualidad es una dimensión básica de las personas y a su vez se demuestra una alta correlación con la salud 
y el bienestar (Kaye y Raghavan, 2002). Los profesionales sociales si quieren ofrecer una atención holística, deben tener en cuenta ineludiblemente la dimensión interior de la persona, sus necesidades no materiales $\mathrm{y}$, por tanto, la vivencia espiritual que cada una tiene.

Estudios centrados en personas con discapacidad intelectual (Swinton, 2002; Kaye y Raghavan, 2002; Clarke y Cardman, 2002) ponen de relieve la profunda espiritualidad, en muchos casos expresada en términos religiosos, que hay en ellas. La idea de Dios, en algunos, es un marco de confianza y de sentido. Fuera del ámbito religioso la idea de amistad también es vista como una fuente de espiritualidad que les ayuda a dar sentido y orientación a la propia vida.

Las comunidades religiosas son un elemento importante en la vida de algunas personas con discapacidad intelectual. Pertenecer a una comunidad, formar parte de un grupo, les da un rol de pertinencia y de posibilidad de relaciones muy apreciados y generadores de sentido profundo. Sin embargo, es cierto que, si actúan de forma excluyente, las comunidades también pueden generar los efectos inversos de frustración y sinsentido.

Clarke y Cardman (2002) destacan tres tipos de creencia clave en personas que viven con una discapacidad. Primero, tener confianza en la vida, sentirse cuidadas a pesar de las dificultades; en segundo lugar, creer en sus posibilidades y confiar en ellas; $\mathrm{y}$, finalmente, encontrar sentido a sus vidas tal como son.

Las residencias de personas con discapacidad son espacios fundamentales en las que tener en cuenta la dimensión espiritual teniendo en cuenta que son los lugares de referencia para estas personas, los lugares donde están viviendo y que deben responder a aspectos que van más allá del cuidado físico o el cobijo.El artículo responde a la pregunta de cuál es la situación de las cuestiones relacionadas con las necesidades no materiales en los entornos residenciales para personas con discapacidad intelectual adultas y lo hace a partir de los resultados de una encuesta y entrevistas que se realizaron al respecto.

\section{METODOLOGÍA}

Se ha construido una encuesta como instrumento de investigación sobre necesidades no materiales en recursos residenciales para personas con discapacidad intelectual. Las preguntas formaban parte de la segunda parte de un cuestionario creado y validado por Vilar y Riberas (2017) en el que se abordaban otras cuestiones.

Para validar el instrumento se constituyó un panel de diez expertos teniendo en cuenta las indicaciones de Hyrkäs et al. (2003) citado por EscobarPérez y Cuervo Martínez (2007, p. 29) que establece este número como el confiable de la "validez de contenido de un instrumento 
Revista científica de la Asociación de Historia y Antropología de los Cuidados (Universidad de Alicante)

(...) para asegurar que el acuerdo se deba al azar”. Para la selección de los validadores se han tenido en cuenta que cumplieran al menos los tres primeros criterios de Skjong y Wentworth (2001, p. 4) citados por Escobar-Pérez y Cuervo Martínez (2007, p. 29).

En cuanto a la fiabilidad del instrumento, se ha calculado el coeficiente alfa de Cronbach ( $\alpha)$ para evaluar la consistencia interna de éste teniendo en cuenta el análisis que hacen Hogan et al. (2000) citados por Soler y Soler (2012). El resultado de 0.77 en el cuestionario completo se ha considerado satisfactorio de igual forma que en el área temática de necesidades no materiales obtiene un valor de 0.89 .

El cuestionario ha sido respondido por un total de 172 profesionales que trabajan en centros residenciales donde viven personas con discapacidad intelectual. Se ha procedido a pasar el cuestionario entre los meses de julio y octubre del año 2016. El análisis estadístico se ha realizado posteriormente hasta febrero del año 2017. El formulario online se distribuyó a profesionales y entidades del sector, sin previa selección, que gestionan residencias para personas con discapacidad intelectual con la ayuda de la Plataforma de Entidades DINCAT-Plena Inclusión. El cuestionario incorporaba el consentimiento informado y la información sobre la investigación. Además, todo el procedimiento metodológico ha superado las validaciones éticas correspondientes por tratarse de una parte de una tesis doctoral (Martínez-Rivera, 2017).

Después del análisis del cuestionario, a modo de triangulación de los datos obtenidos, se realizaron cuatro entrevistas a familiares, a cuatro profesionales con más de diez años de experiencia y también a cuatro personas con discapacidad intelectual (entre 45 y 70 años) que viven en este tipo de recursos residenciales desde hace más de diez años.

\section{RESULTADO Y DISCUSIÓN}

El cuestionario aborda las posibilidades que tienen las personas con discapacidad de decidir sobre ellos mismos, así como las posibilidades que tienen en el entorno residencial de pensar o meditar sobre ellos mismos. Los datos obtenidos se han dividido entre aquellos que hacen referencia a recursos residenciales permanentes y a los que son proyectos encaminados a la autonomía en el propio hogar de las personas. Se ha considerado oportuno hacer esta diferenciación teniendo en cuenta que hay diferencias importantes entre los grados de autonomía, siendo las personas de residencias permanentes las que necesitan más apoyos.

Los profesionales que trabajan en recursos residenciales permanentes obtienen 2.44 de puntuación media sobre unas valoraciones que van del 1 al 4 . El recurso 
residencial como espacio de silencio es el valor más elevado junto con el que se refiere a que los residentes deciden sobre ellos mismos además de la percepción de espacio de tiempo y silencio personal. La puntuación más elevada se da en la que se refiere a la relación de la residencia como espacio de silencio y es valorado como que se da muchas veces en un $24.8 \%$ de los encuestados.

La posibilidad de tomar decisiones sobre uno mismo es una de las necesidades no materiales más importantes (Clarke y Cardman, 2002). A la pregunta sobre ésta, las decisiones a corto, medio y largo plazo, las puntuaciones son cada vez más bajas cuanto más lejano en el tiempo es el impacto de la decisión: 2.53, 2.25 y 2.05 puntos. Esto quiere decir que tienen más participación

sobre sus propias decisiones cuanto a más corto plazo son. Cuando en la investigación se pregunta sobre si el recurso residencial podría ser un espacio donde las personas con discapacidad decidan sobre su propia vida el porcentaje que indica que lo hacen bastante o mucho es del $63.6 \%$, aunque el peso del mucho solo es del $12.7 \%$ (véase figura 1 y 2).

Por otro lado, independientemente de si las decisiones que toman las personas usuarias sobre aspectos de su vida son de fuera del servicio, éstas, solo se toman mucho en un $10.1 \%$ de las veces, aunque son más que las que se toman muchas veces sobre el propio servicio, que solo son el
$5.5 \%$. Además, en el otro extremo de respuesta, los profesionales dicen que nunca o pocas veces toman decisiones sobre aspectos de fuera del servicio en un 56.9\% y si son del servicio nunca lo hacen o poco en un $71.8 \%$ de las veces. Esto se traduce en que, de media, cuando hay que puntuar si deciden sobre el propio servicio se valora con un 2.29 y sobre aspectos de fuera del servicio se eleva hasta el 2.56 .

Por otro lado, según los profesionales, existe una baja participación en las residencias permanentes en actividades relacionadas con la religión en comparación con el resto de ítems. Pero hay que tener en cuenta que un 20.0\% afirman que existe bastante práctica en este sentido y un $1.8 \%$ dicen que se da mucho (Figura 1)

Por otro lado, en los recursos residenciales de apoyo en el propio hogar se observa un claro aumento en todas las valoraciones de todos los ítems respecto a residencias permanentes, a excepción del que hace referencia a la práctica de actividades religiosas que disminuye. Destaca que llega hasta el 50\% la valoración del recurso como espacio de silencio, situándose en más del doble que en la residencia permanente. Como lugar de tiempo personal aumenta más del doble la valoración y llega hasta el 44.4\%.

En este recurso residencial se ha pasado a afirmar en un $47.4 \%$ que sucede con mucha frecuencia que las personas con discapacidad deciden por ellas mismas, 
Revista científica de la Asociación de Historia y Antropología de los Cuidados (Universidad de Alicante)

dejando atrás el 12.7 del otro recurso. Continúa sucediendo que las personas atendidas deciden más sobre aspectos que pasan fuera del propio servicio que no aquello que tiene que ver con él. En concreto, se valoran las decisiones sobre aspectos externos con un 3.00 y con un 2.82 aquellos que tienen que ver con el servicio. Esta tendencia también se daba en los recursos residenciales de estancia permanente (Figura 2).

Tanto en un tipo de recurso como el otro se da un patrón casi idéntico en la disminución lineal de la decisión de las personas que viven allí en cuanto a aspectos a corto, medio y largo plazo. Siempre hay que tener en cuenta que en el recurso de apoyo en el propio hogar se sitúa por encima del de residencia permanente. En este tipo de dispositivos sociales se espera fundamentalmente que se potencie el autogobierno. Y este hay que valorarlo teniendo en cuenta que la autonomía de la persona también consiste en la planificación de su vida más allá de las decisiones cotidianas (o a corto plazo) que en muchas ocasiones pueden parecer menos relevantes para el sentido vital de las personas (Figura $3)$.

Con el gráfico comparativo de las medias (véase figura 4) podemos apreciar ese aumento claro en todos los ítems de este bloque sobre necesidades no materiales cuando nos referimos a un recurso residencial de apoyo en el propio hogar. Por otro lado, se destacan las tres preguntas que hacen referencia a las decisiones a corto, medio y largo plazo donde se observa esa diferencia entre un recurso y otro de forma casi proporcional (Figura 4).

Los comentarios abiertos en el cuestionario, por parte de profesionales, admiten mayoritariamente que sería interesante poder trabajar de manera más explícita estas cuestiones. Hay otras respuestas que comparten que hacen actividades relacionadas con las necesidades no materiales sobre las que se les pregunta. De nuevo, en algunas ocasiones se hace hincapié en los beneficios que supondría poder tener recursos con habitaciones únicamente individuales y los beneficios que supondría en los usuarios. Hay otro bloque de comentarios abiertos que hablan de la falta de recursos o tiempo en poder trabajar aspectos que vayan más allá de lo más asistencial.

En las entrevistas a los familiares ha habido una aceptación unánime de que estas necesidades las tienen todas las personas y que por lo tanto las personas con discapacidad intelectual también. En cuanto a aspectos que hacen referencia a la protección que supone un espacio como es el de la casa de una persona, todas las personas han manifestado que su familiar sí que siente la vivienda tutelada como un espacio de seguridad. La confortabilidad depende de cada persona, pero en general se está de acuerdo que el espacio de residencia sí que lo es. En gran medida se manifiesta que el familiar siente como casa propia la 
residencia, aunque se admite que el hecho de compartir la vida con más personas genera cierta dificultad.

En algunos de los comentarios, los familiares manifiestan un beneficio importante en el hecho de haber empezado a vivir en la vivienda tutelada. En algunos casos se expresa que este paso ha sido generadora de conciencia personal puesto que anteriormente las madres (así se expresa) han sido especialmente protectoras y ello no facilitaba la independencia y autonomía de los hijos. Algunas de las afirmaciones más importantes, en este sentido, fueron que "Desde que faltan mis padres mi hermano vive más la vida o la conoce más porque mis padres eran mayores y no lo podían sacar a ningún sitio” y “en calidad de vida ha mejorado mucho" (refiriéndose al hecho de vivir en el centro residencial. Relacionando la capacidad de decisión con la autonomía, una de las familiares también decía que "él estaba como un señor sin esforzarse" (refiriéndose a cuando vivía con los padres) "y en los pisos tiene que esforzarse (...) se ha tenido que espabilar, y eso es positivo"

Por otro lado, en las entrevistas a los profesionales los argumentos se centran más en los aspectos que se podrían mejorar, a diferencia de los familiares que centraron mucho el discurso en lo que consideran que ha supuesto a sus familiares estar en el recurso residencial. De todas formas, los profesionales saben focalizar en qué dirección hace falta esforzarse para generar esa mejora. Los profesionales admiten que las personas usuarias deberían tener más espacios de participación sobre la organización del recurso. Además, también se hace referencia a que debería mejorarse el apoyo o el acompañamiento para que pudieran decidir no solamente sobre el recurso sino sobre lo que quieren hacer en la vida. Admiten que ha habido mucha mejora al respecto pero que, especialmente las personas con menos capacidades, no tienen todos los espacios que se desearían. Esta mejora en cuanto al poder de decisión de las personas que viven en los recursos pasa por intentar individualizar al máximo la atención, aunque también se admite que algunas veces no es fácil por los recursos de que se disponen.

La evolución de los recursos se ve como bastante clara durante los últimos años y se especifica que, si hay alguna persona que hace una demanda de poder estar solo, pensar, estar en silencio, pasear, etc. se permite que lo haga sin dudarlo. En este sentido se ha procurado, especialmente en los últimos años, que puedan gozar de espacios personales e individuales de los que ha hecho demanda cada persona de una manera determinada. Esto también ha supuesto realizar un trabajo profesional donde se dirigen menos las actividades.

Los profesionales están de acuerdo en que las personas con diversidad funcional adultas han estado menos acostumbradas a tener que decidir y en general les cuesta más. Se tiene la sospecha de que las nuevas 
Revista científica de la Asociación de Historia y Antropología de los Cuidados (Universidad de Alicante)

generaciones, las personas más jóvenes están ejerciendo un papel de más protagonismo sobre su vida.

En las entrevistas a las personas usuarias se han detectado determinadas prácticas para pensar o meditar que tienen que ver con permanecer en las habitaciones a ser posible solos. Pero también hay otro tipo de rituales como pueden ser pasear por el barrio o bien, en un par de casos, asistir a la iglesia sin que eso represente necesariamente participar del ritual de alguna ceremonia como ir a Misa. Se asocia, esta alternativa, a la idea de silencio y de recuerdo de personas importantes para su vida y ya fallecidas. Han surgido bastantes narraciones donde la tranquilidad y la posibilidad de estar con uno mismo se busca en espacios del entorno y no en la propia vivienda. En otros casos, establecen estrategias para encontrar esa tranquilidad: “después de cenar me gusta venir aquí (a la habitación) (...), miro por la terraza (..) al mar (el recurso está delante del mar), si hace buen día salgo fuera (a la calle). Otra de las personas usuarias reconoce que la tranquilidad la encuentran "escuchando música con los cascos (auriculares) (y señalando su cama como el lugar donde la escucha). Además, también coincide que encuentra tranquilidad cuando "se va a dar una vuelta el viernes”. Pero también hay alguna persona usuaria que afirma que el sitio más tranquilo es "en casa de mi hermana” a la vez que confirma que su casa es el centro residencial. En las entrevistas a familiares se reflejaba cuando afirmaban que "eso lo hace mucho desde que mi madre murió. (...) Y no será porque nosotros fuéramos a misa ni fuésemos cristianos ni rezáramos” (...) "que nadie le moleste, se siente, no sé, quizás realizado. Ese silencio espiritual le llena”

Por lo que hace referencia a aspectos que tienen que ver con la planificación de la propia vida, las personas usuarias no han manifestado ideas generales sobre deseos que tengan que ver con la planificación de su vida. Cuando se ha planteado esta cuestión ha sido complicado poder llegar hasta alguna propuesta general y en algún caso ha habido manifestación de alguna demanda más bien concreta. También es verdad que algunas de las respuestas sobre la propia actividad llevan implícitamente una dependencia de los profesionales para poder realizar algunas actividades personales. Estas cuestiones son expresadas desde la comodidad de poder, en cierta medida, pedir permiso sabiendo que nadie lo impedirá.

Se detectan correlaciones significativas positivas en lo que se refiere a la mayor parte de necesidades no materiales cuando los profesionales afirman que las personas usuarias pueden decidir sobre el recurso residencial. Se da en los dos tipos de recursos tanto permanentes como los de apoyo. Esto quiere decir que a más posibilidad de participación más sucede que se valoran como cubiertas estas necesidades. 
Si los profesionales de recursos residenciales de estancia permanente creen que podrían participar más los que no lo hacen, también aumenta la cobertura de las necesidades no materiales en la mayoría de los ítems analizados.

$\mathrm{Si}$ analizamos las correlaciones referentes a las necesidades no materiales encontramos que se dan muchas de carácter negativo significativas especialmente con los conflictos entre profesionales que se analizaron para otra investigación. Es decir que a más cobertura de las necesidades no materiales se da que hay menos conflictos éticos (Vilar, 2013; Vilar y Riberas, 2017). Estadísticamente no demuestra que uno sea causa del otro, pero sí que se dan estas relaciones. Ocurre de una forma similar en los recursos con apoyo, pero centrado especialmente en las necesidades no materiales que tienen que ver con el silencio, la posibilidad de pensar o meditar.

Sobre el aspecto que hace referencia a los conflictos con las personas atendidas se produce correlación negativa y significativa en la mayoría de los ítems cuando analizamos tanto las necesidades no materiales y las agresiones como fuente de conflicto en los centros residenciales permanentes, así como en las necesidades no materiales y los conflictos con las respuestas justas en los recursos de apoyo en la vivienda. La investigación puede entenderse como una base sobre la que se podrían realizar más entrevistas en profundidad tanto a profesionales y familiares como a personas con diversidad funcional intelectual. Esta fase de investigación podría aportar muchos matices que, aunque esta investigación los ha revelado en parte, se podrían profundizar.

Por otro lado, la investigación se hace bajo un contexto social de Estado de Bienestar en el que existen políticas sociales destinadas a personas con diversidad funcional desde hace décadas, pero el estudio podría relevar interesantes comparaciones con otros contextos sociopolíticos diferentes. Además, la comparación sería también de mucha utilidad en políticas sociales si se realizara basada en personas con las mismas características, pero en otros contextos desinstitucionalizados mediante asistencia personal (González-Rodríguez, VerdeDiego y Pérez-Lahoz, 2019).

\section{CONCLUSIONES}

Los recursos residenciales para personas con discapacidad intelectual deben satisfacer necesidades que van más allá de las de protección y cobijo. Es por eso que en el proceso de investigación los profesionales que trabajan con personas con discapacidad admiten interés en poder hacer explícito el abordaje en relación a las necesidades no materiales en los centros residenciales.

Las personas con discapacidad, independientemente del tipo de recurso residencial donde viven, toman menos decisiones sobre sus propias vidas cuanto más a largo plazo son estas decisiones. Es 
Revista científica de la Asociación de Historia y Antropología de los Cuidados (Universidad de Alicante)

decir, están reforzadas las decisiones que tienen que ver más con lo inmediato.

En el caso de las personas que viven en proyectos de autonomía en el propio hogar toman substancialmente más decisiones sobre la propia casa donde viven en comparación a las personas de residencias. En ambos casos las decisiones que toman hacen referencia mucho más a sus propias vidas que no en relación al lugar donde están viviendo.

Tanto las residencias como los proyectos de autonomía son percibidos con bastante o mucha posibilidad de espacios de silencio. En este caso, en los proyectos de autonomía en el propio hogar la percepción de que en el lugar se da mucho la situación de espacio de silencio se dobla respecto a las residencias y llegan a la mitad de las respuestas que dan los profesionales.

Las actividades relacionadas con la religión no son mayoritarias en ambos tipos de recursos, aunque, uno de cada cinco profesionales admite bastante o mucha actividad al respecto.

En general todas las cuestiones referidas a las necesidades no materiales tienen una tendencia a ser más valoradas en los recursos de apoyo en el hogar. Pero es importante remarcar que la investigación nos lleva a pensar que todos los recursos residenciales en general proporcionan la posibilidad de que sean espacios de silencio y encuentro con uno mismo. En este sentido cabe plantearse si es resultado del azar o si hay mecanismos sistematizados para que esta necesidad pueda ser satisfecha por un planteamiento explícito en los objetivos de los proyectos.

En general, el trabajo individualizado en los recursos para personas con diversidad funcional intelectual permite la posibilidad de acompañar en la toma de decisiones. Esto, a su vez, potencia la generación de una vida independiente donde la construcción personal del futuro inmediato, pero también a largo plazo, es importante de la misma forma que lo es para cualquier persona.

\section{BIBLIOGRAFIA}

Clarke, K. y Cardman, F. (2002). Spiritual Resilience in People Who Live Well with Lifelong Disability. Journal of Religion, Disability \& Health, 6(1), 23-36. DOI: https://doi.org/10.1300/J095v06n01 03

Escobar-Pérez, J. y Cuervo, A. (2007). Validez de contenido y juicio de expertos: una aproximación a su utilización. Avances en Medición, 6, 27-36.

Girard, G. (2007). Espiritualidad: ¿promueve resiliencia? En Adolescencia y resiliencia. Buenos Aires: Paidós.

González-Rodríguez, R., Verde-Diego, C. \& Pérez-Lahoz, V. (2019). La asistencia personal como nuevo derecho de ciudadanía: una mirada desde el ámbito de los profesionales de la salud. Cultura de los Cuidados (Edición digital), 23(53). Recuperado en: http://dx.doi.org/10.14198/cuid.2019.53.11

Jaramillo-Vélez, D., Ospina-Muñoz, D., Cabarcas-Iglesias, G., y Humphreys, J. 
(2005). Resiliencia, Espiritualidad, Aflicción y Tácticas de Resolución de Conflictos en Mujeres Maltratadas. Revista de Salud Pública, 7(3), 281-292. Disponible en:

\section{http://redalyc.org/articulo.oa?id=42270304}

Kaye, J., y Raghavan, S. K. (2002). Spirituality in Disability and Illness. Journal of Religion and Health, 41(3), 231-242. DOI:

https://doi.org/10.1023/A:1020284819593

Koening, H.G. (2001). Handbook of religion and health. Nueva York: Oxford University Press.

Martínez-Rivera, O. (2017). El recurso residencial como posibilidad para vivir $y$ trabajar en él (Tesis no publicada). Universidad de Barcelona: Barcelona.

Organización Mundial de la Salud (1998). Manual de instrucciones de la OMS sobre calidad de vida. Ginebra: OMS.

Otón, J. (2018). Interioridad $y$ espiritualidad. Bilbao: Sal Terrae.

Senreich, E. (2013). An inclusive definition of spirituality for Social Work education and practice. Journal of Social Work Education, 49, 548-563. DOI: https://doi.org/10.1080/10437797.2013.812 $\underline{460}$

Soler y Soler (2012). Usos del coeficiente alfa de Cronbach en el análisis de instrumentos escritos. Revista Médica Electrónica, 34 (1), 01-06. Disponible en http://scielo.sld.cu/scielo.php?script=sci_art text\&pid=S1684-18242012000100001

Swinton, J. (2002). Spirituality and the Lives of People with Learning Disabilities. Tizard Learning Disability Review, 7(4), 29. DOI: https://doi.org/10.1108/1359547420020003 7
Vanistendael, S. (2003). Resiliencia y

espiritualidad. Ginebra: Bice.

Vilar, J. (2003). El tractament dels confliectes ètics en la pràctica profesional: aproximació a una ética aplicada. En: Planella, J.; Vilar, J. L'educació social: projectes, perspectives i camins. 195-216. Barcelona: Pleniluni.

Vilar, J., y Riberas, G. (2017). Tipos de conflicto ético y formas de gestionarlos en la educación social y el trabajo social. Retos en las políticas de formación. Archivos Analíticos de Políticas Educativas, 25(52). Disponible en: http://www.redalyc.org/articulo.oa?id=2750 50047052 
Figura 1. Distribución de necesidades no materiales en residencias permanentes

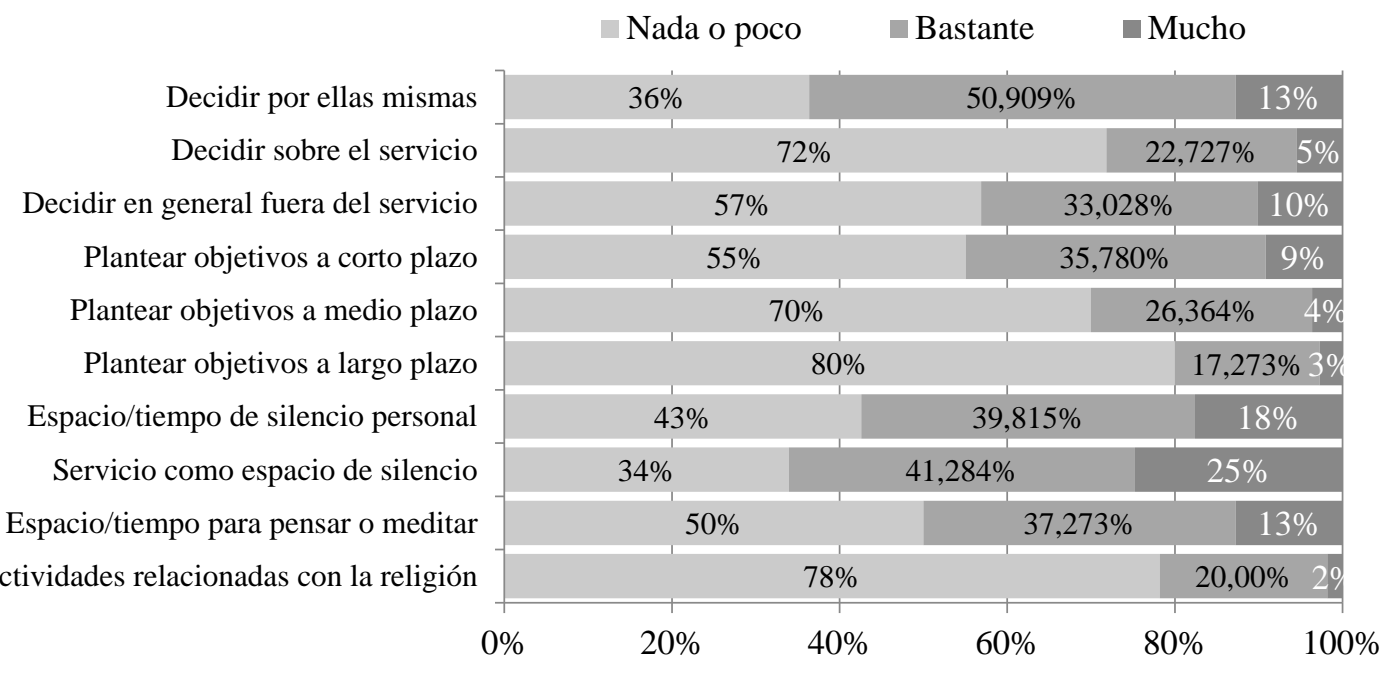

Figura 2. Medias de las necesidades no materiales en residencias

Media $\quad$ Media total $(2,44)$

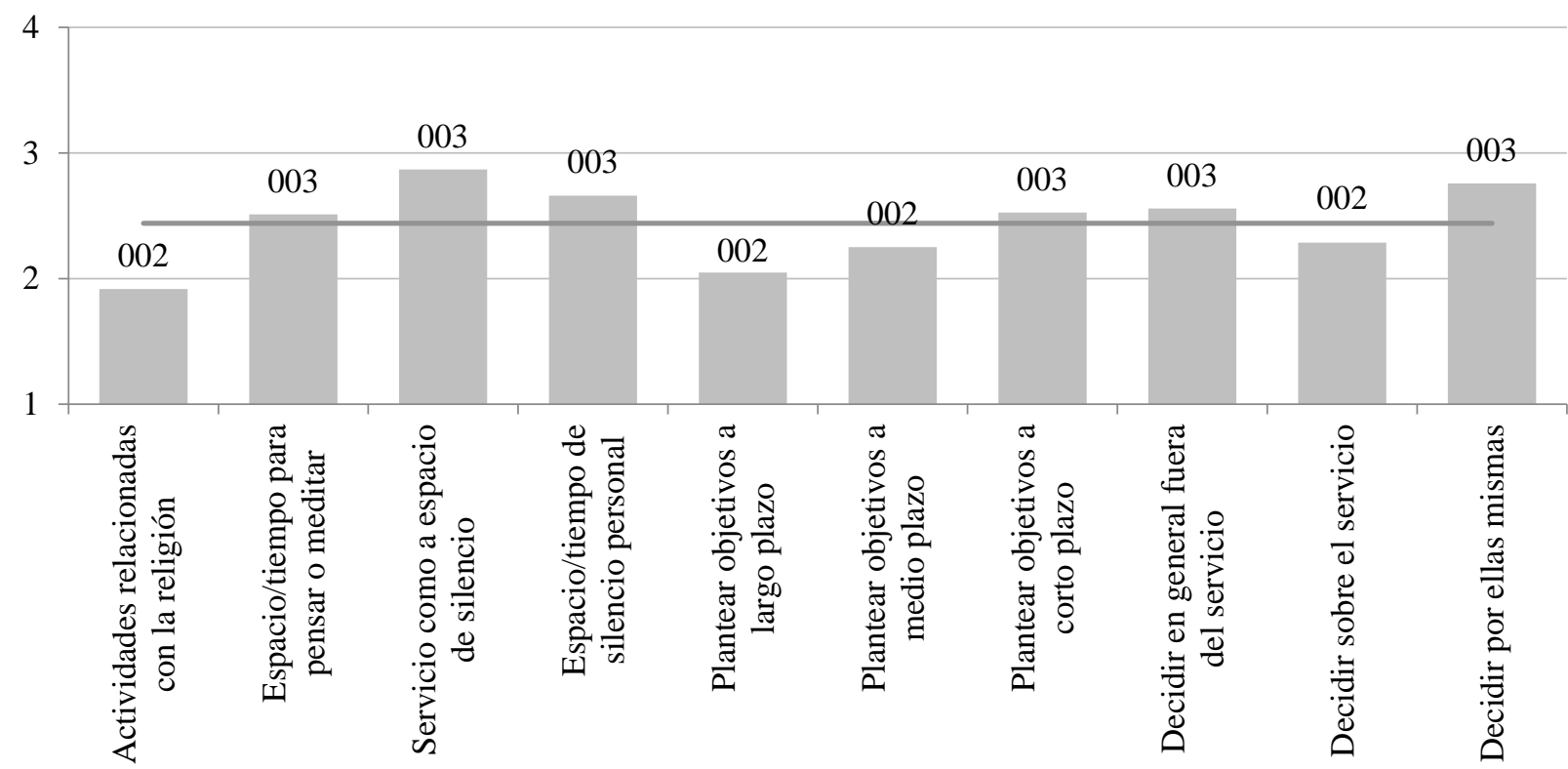


Figura 3. Distribución de necesidades no materiales en apoyo en el hogar

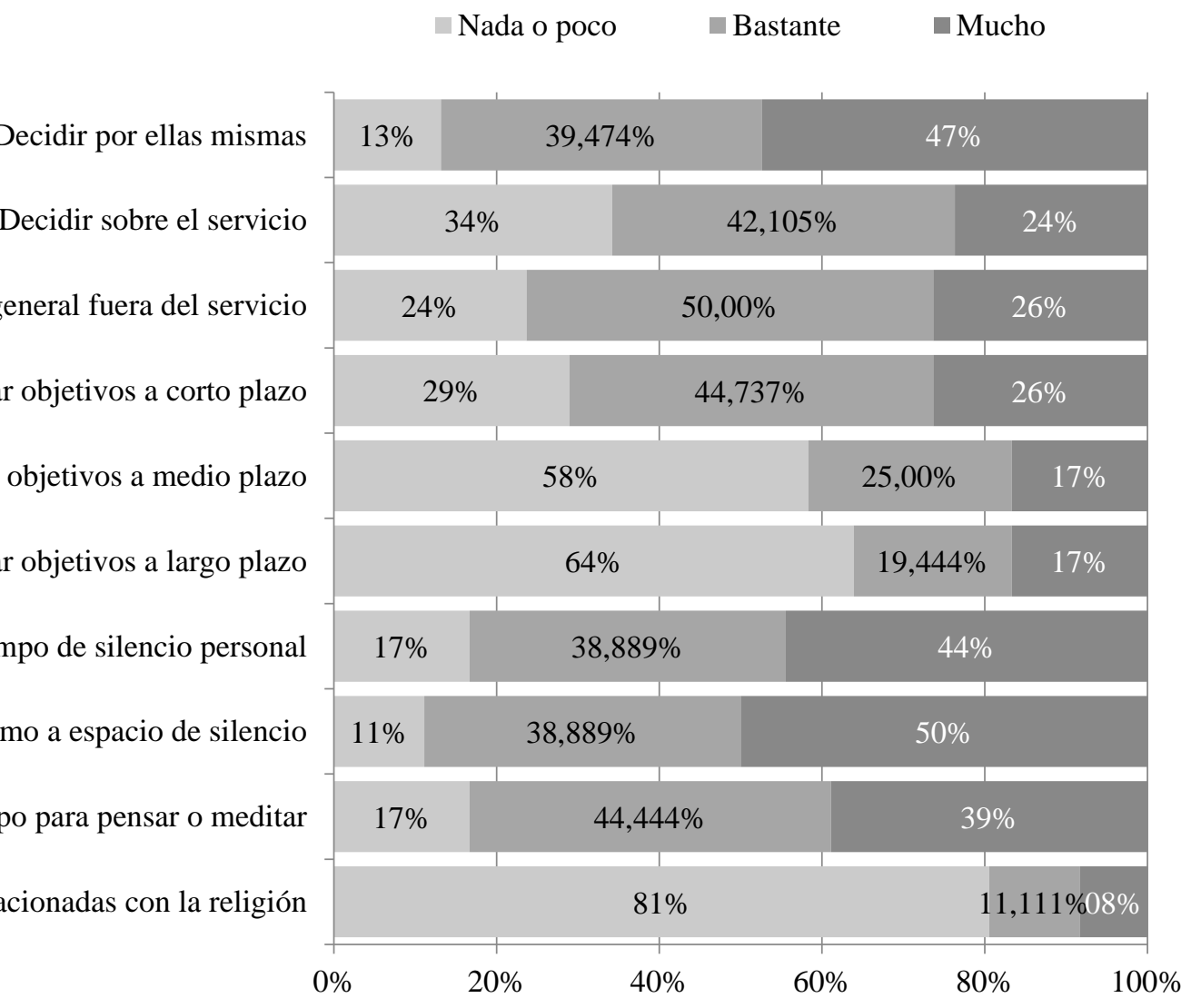


Figura 4. Medias de necesidades no materiales en residencias y apoyo en el hogar

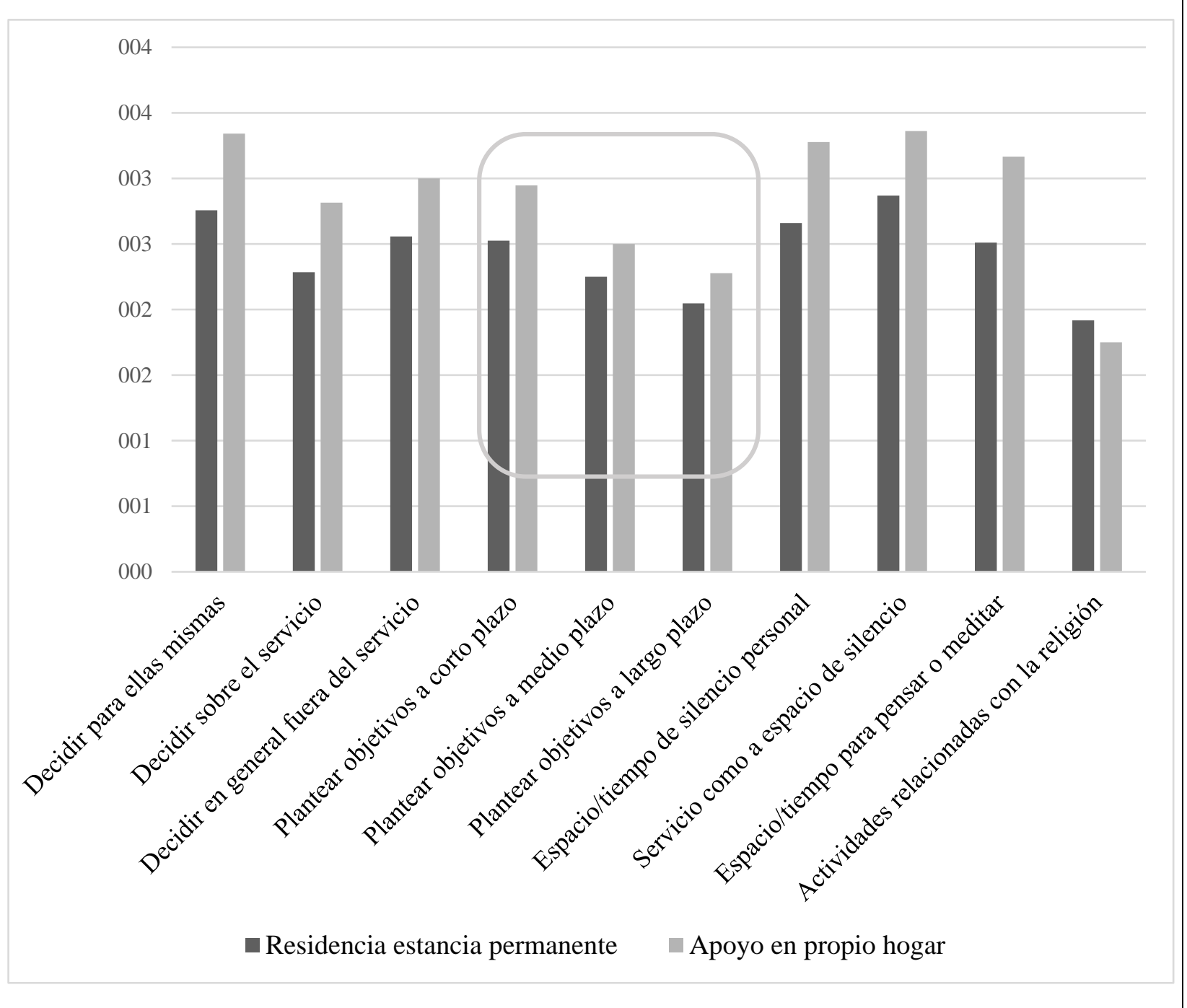

